\title{
Diagnostic and Therapeutic Potential of Extracellular Vesicles in B-Cell Malignancies
}

\author{
Ernesto Gargiulo ${ }^{1}$, Pablo Elías Morande ${ }^{1,2}$, Anne Largeot ${ }^{1}$, Etienne Moussay ${ }^{1 * \dagger}$ and \\ Jérôme Paggetti ${ }^{1 * t}$
}

${ }^{1}$ Tumor-Stroma Interactions, Department of Oncology, Luxembourg Institute of Health, Luxembourg, Luxembourg, ${ }^{2}$ Instituto de Medicina Experimental (IMEX)-CONICET-Academia Nacional de Medicina, Buenos Aires, Argentina

Extracellular vesicles (EV), comprising microvesicles and exosomes, are particles released by every cell of an organism, found in all biological fluids, and commonly involved in cell-to-cell communication through the transfer of cargo materials such as miRNA, proteins, and immune-related ligands (e.g., FasL and PD-L1). An important characteristic of EV is that their composition, abundance, and roles are tightly related to the parental cells. This translates into a higher release of characteristic pro-tumor EV

OPEN ACCESS

Edited by:

Cyrus Khandanpour, University Hospital Münster, Germany

Reviewed by:

Shaji Kumar

Mayo Clinic, United States

Ingo Ringshausen,

University of Cambridge,

United Kingdom

*Correspondence:

Jérôme Paggett

jerome.paggetti@lih.lu

Etienne Moussay

etienne.moussay@lih.lu

tThese authors share senior authorship

Specialty section:

This article was submitted to Hematologic Malignancies,

a section of the journal

Frontiers in Oncology

Received: 07 July 2020 Accepted: 04 September 2020 Published: 29 September 2020

Citation:

Gargiulo E, Morande PE Largeot A, Moussay E and Paggetti J (2020) Diagnostic and Therapeutic Potential of Extracellular Vesicles

in B-Cell Malignancies.

Front. Oncol. 10:580874. doi: 10.3389/fonc. 2020.580874 by cancer cells that leads to harming signals toward healthy microenvironment cells. In line with this, the key role of tumor-derived EV in cancer progression was demonstrated in multiple studies and is considered a hot topic in the field of oncology. Given their characteristics, tumor-derived EV carry important information concerning the state of tumor cells. This can be used to follow the outset, development, and progression of the neoplasia and to evaluate the design of appropriate therapeutic strategies. In keeping with this, the present brief review will focus on B-cell malignancies and how EV can be used as potential biomarkers to follow disease progression and stage. Furthermore, we will explore several proposed strategies aimed at using biologically engineered EV for treatment (e.g., drug delivery mechanisms) as well as for impairing the biogenesis, release, and internalization of cancer-derived EV, with the final objective to disrupt tumor-microenvironment communication.

Keywords: extracellular vesicles, exosome, CLL, leukemia, lymphoma, myeloma, EV-based therapy

\section{INTRODUCTION}

Extracellular vesicles (EV) are vesicular components released by every cell of an organism. This broad group consists of small EV or exosomes (sEV; 30-150 nm) characterized by an endocytic origin, microvesicles (MV; $100 \mathrm{~nm}-5 \mu \mathrm{m}$ ) shed from the plasma membrane, and apoptotic bodies $(<5 \mu \mathrm{m})$ derived from membrane disintegration (1). The study of EV is a process in constant

Abbreviations: ALL, acute lymphocytic leukemia; ALV, artificial lipid vesicles; BTK, Bruton tyrosine kinase; CLL, chronic lymphocytic leukemia; ctDNA, circulating tumor DNA; CTX, cyclophosphamide; DC, dendritic cell(s); DLBCL, diffuse large B-cell lymphoma; EBV, Epstein-Barr virus; EGFR, epidermal growth factor receptor; EV, extracellular vesicle(s); HL, Hodgkin lymphoma; HM, hematological malignancy; HS, heparan sulfate; ICP, immune checkpoint; IFN- $\gamma$, interferon- $\gamma$; IL6ST, interleukin 6-signal transducer; MDR, multiple drug resistance; MSC, mesenchymal stem cell(s); MM, multiple myeloma; MV, microvesicle(s); NK, natural killer(s); NV, nanoparticle(s); PG, proteoglycans; sEV, small extracellular vesicle(s); shRNA, small hairpin RNA; SIRP $\alpha$, signal regulatory protein $\alpha$; SMART, synthetic multivalent antibodies retargeted; TGF- $\beta(1)$, transforming growth factor- $\beta(1)$; TNFR1, tumor necrosis factor receptor 1 . 
evolution, where EV characteristics, as well as nomenclature and isolation, are in continuous improvement (2-5).

Extracellular vesicles can be virtually found in every biofluid, which makes them relatively easy to recover and analyze (6). In the early years of EV-focused research, their synthesis and release were considered as a mechanism to remove harming material from the cell (7). Nowadays, this notion has been replaced by considering the mechanism as an active way to transfer material to targeted cells (1). Indeed the most common and accepted role of EV is intercellular communication through ligand-receptor interactions and the transfer of molecular cargoes.

The composition of EV is extremely heterogeneous. Depending on their nature, origin, physiological context, and parental cells, EV contain and transfer distinct elements to targeted cells, such as proteins, nucleic acids, lipids, metabolites, and organelles (8-10). The interest in understanding the role of $\mathrm{EV}$ in cancer is due to their unique ability to reeducate and attenuate the normal activity of healthy cells across the whole body. In line with this, B-cell malignancyderived EV have been shown to interact with the surrounding microenvironment, leading to its profound remodeling. EVbased communication assists cancer development by stimulating tumor cell proliferation and migration (11), by modifying distant microenvironment to generate a pre-metastatic niche (12), and by strategically inhibiting tumor immune surveillance and anti-tumor response $(13,14)$.

\section{EV DIAGNOSTIC AND THERAPEUTIC POTENTIAL}

Due to a complex and parental-cell-dependent molecular cargo and to their presence in every biological fluid, EV represent an innovative tool for the design of diagnostic and therapeutic strategies in B-cell malignancies. In the sections below, we will explore the multiple potential uses of EV: (i) EV as biomarkers, (ii) EV as therapeutic targets, (iii) EV in immune evasion and use in immunotherapy, and (iv) biologically engineered EV (Figure 1 and Table 1).

\section{EV as Biomarkers}

Given that EV are continuously released by all the cells and that they can be recovered from every fluid in the body, they clearly represent a potential source of biomarkers (15, 16). Compared with most common biomarkers obtained from liquid biopsies, such as circulating tumor DNA (ctDNA), EV represent a more reliable source of information and can be easily incorporated in the diagnostic routine. Specifically, ctDNA represents only a minimal fraction of plasma-cell-free DNA $(17,18)$; thus, despite its high specificity, the starting amount is already challenging $(17,19)$. Furthermore, ctDNA is often affected by high fragmentation and low stability $(20,21)$. EV are very stable and, depending on the time of use, can be stored at different temperatures for extended periods of time (several weeks at $4^{\circ} \mathrm{C}$, several months at $-80^{\circ} \mathrm{C}$, and several years in liquid nitrogen) (22). Cancer cells generally produce high amounts of EV that mirror the characteristics-as a sort of fingerprint-of the parental cancer cells (23-25). Indeed cargo composition (e.g., microRNA and proteins) and transmembrane molecules (tumor antigens or immune cell markers) allows the identification of the parental cell and its physiological state (24, 26). Analysis of EV isolated from patients was confirmed to be an important tool in the detection of cancer development at an early stage and to stratify patients based on intrinsic tumor characteristics and for following patients in remission (16, 27, 28).

In line with this, we have shown that the plasma of a patient affected by chronic lymphocytic leukemia (CLL) is highly enriched in multiple key microRNAs (e.g., miR-21, miR-155, and miR-146a) which can potentially affect multiple pathways involved in the pathogenesis and the progression of the disease. MicroRNA expression profile can also be used to stratify CLL patients in view of a more personalized treatment and follow-up (29). Importantly, we and others found these same microRNAs and some additional ones (miR-148a and Let$7 \mathrm{~g}$ ) also in CLL-derived EV, demonstrating how these vesicles can indirectly provide information on cellular state $(30,31)$. Van Eijndhoven and colleagues detected a higher level of EV carrying miR-24-3p, miR-127-3p, miR-21-5p, miR155-5p, and Let7a-5p in the plasma of Hodgkin lymphoma patients compared with controls. Upon treatment, patients showing remission had a stable reduction of these miRNAs compared with relapse patients who displayed their progressive accumulation (32). Interestingly, a further study performed on a large multiple myeloma (MM) cohort described that the progression-free survival and the overall survival of patients could be successfully followed by monitoring the plasma levels of miR-18a and Let7b, which were found to be highly enriched in MMderived EV (33).

Beyond miRNA content, RNA packed in plasma EV has the potential to be used to define tumor mutation landscape, such as BRAF V600E for melanoma, with the aim to detect early-stage disease or define the best clinical approach for certain patients (34). This strategy may be transposed to mutated RNA contained in circulating EV from B-cell malignancies, with the attempt to perform a personalized patient follow-up and therapy.

EV reflect parental cell also through the presence of cellular markers on the EV membrane and in their protein cargo (35). Different studies show that CLL-derived EV from a patient's plasma present typical markers of leukemic cells (e.g., CD19, CD20, CD5, and CD37) and major histocompatibility complex (e.g., HLA-A, HLA-B, and HLA-C) $(36,37)$. Despite this correlation, it is essential to highlight that not all markers or their possible combinations are always present on EV released by tumor cells. Indeed CLL cells can release a subset of EV carrying CD52 but only few CD19 molecules; this particular marker combination was shown to be characteristic of CLL patients with advanced disease (38). Another study by Harshman and colleagues showed the presence of typical MM cell (CD38, CD138, and CD147) and treatment resistance (CD44)-associated markers on MM-derived EV isolated from a patient's serum $(39,40)$.

In sum, EV represent a faithful snapshot of the parental cell that synthesized them. The follow-up of proteins 


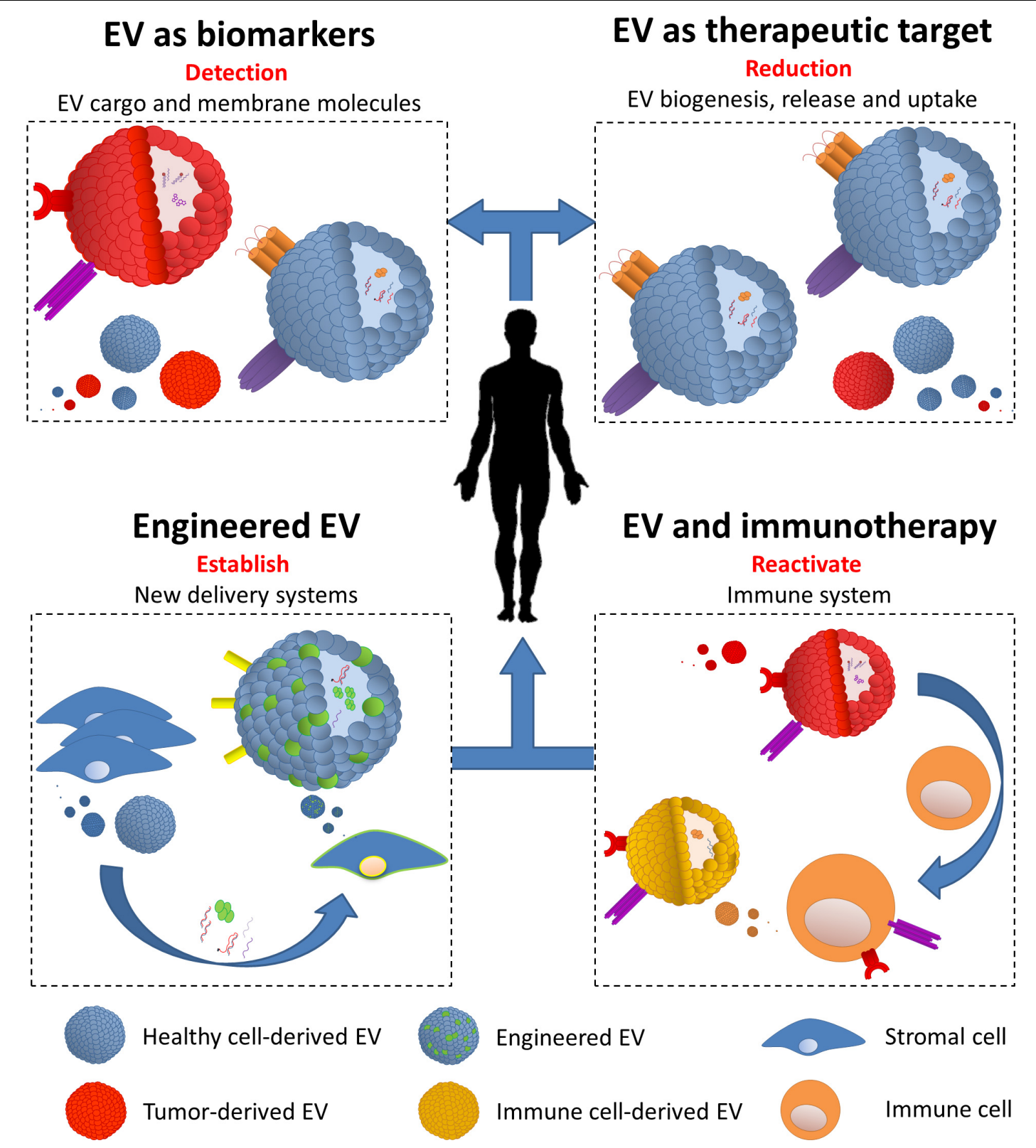

FIGURE 1 | Extracellular vesicles (EV) diagnostic and therapeutic potential. (i) EV as biomarkers: multiple physiological characteristics allow EV to be easily collected from different biological sources (e.g., plasma and urine). Definition of content and carried molecules is essential to determine their origin, thus detecting tumor in different stages of the disease and in post-treatment follow-ups. (ii) EV as therapeutic targets: biogenesis, release, and uptake are all key steps which can be targeted in order to decrease the circulating amount and the function of tumor-derived EV. Beyond this, targeting active EV components (e.g., immune checkpoint) decreases their impact on various aspects on targeted cells. (iii) EV and immunotherapy: tumor cells have the ability to re-educate the immune system via the release of EV, thus able to affect the function of multiple immune cells. Despite this, tumor-derived EV can be strategically used to build a strong immune response by the endogenous immune cells (e.g., dendritic cells) and use their EV to diminish cancer cell functions. (iv) Engineered EV: together with nanoparticles, EV can be modified to carry specific molecules (e.g., shRNA and chemotherapy drugs) and directed to cancer and its microenvironment cells. Engineered EV can be produced by transformed cell lines (e.g., mesenchymal stem cells) and directed against tumor cells.

and RNAs carried by circulating EV in B-cell malignancy patients constitutes a promising source of predictive biomarkers. The low invasiveness of their isolation could allow regular sampling, and by monitoring $\mathrm{EV}$ content, the evolution of tumors within patients can be followed, either before or after anti-cancer treatment, allowing clinicians to select the best course of action for each specific case.

\section{EV as Therapeutic Targets}

Biogenesis, release, and internalization of EV are tightly regulated processes that involve multiple putatively targetable protein 
TABLE 1 | Overview of the diagnostic and the therapeutic potentials of extracellular vesicles in B-cell malignancies.

\begin{tabular}{|c|c|c|c|}
\hline EV Role & Target & B-Cell malignancy & References \\
\hline \multirow[t]{5}{*}{ Biomarker } & miR-21, miR-155, miR-146a, miR-148a, and Let-7g & CLL & $(29-31)$ \\
\hline & miR-24-3p, miR-127-3p, miR-21-5p, miR155-5p, and Let7a-5p & $\mathrm{HL}$ & $(32)$ \\
\hline & miR-18a and Let7b & MM & (33) \\
\hline & CD19, CD20, CD5, CD37, CD52, and MHC & CLL & $(36,37)$ \\
\hline & CD38, CD138, CD147, and CD44 & $\mathrm{MM}$ & $(39,40)$ \\
\hline \multirow[t]{3}{*}{ Target } & EV release (indomethacin) & DLBCL & $(42)$ \\
\hline & EV uptake (low molecular weight heparin) & CLL & $(30)$ \\
\hline & TGF- $\beta 1$ (shRNA) & ALL & $(44,71)$ \\
\hline \multirow[t]{2}{*}{ Immune modulator } & T lymphocytes (DC-derived EV) and DC (poly I:C) & ALL & $(58)$ \\
\hline & TGF- $\beta 1$ (shRNA) & ALL & $(44,71)$ \\
\hline \multirow[t]{2}{*}{ Vaccination } & DC (DLBCL-derived EV) & DLBCL & $(78)$ \\
\hline & T lymphocytes (DC-derived EV) & ALL & $(64)$ \\
\hline \multirow[t]{4}{*}{ Carrier } & c-Myc (siRNA) & Lymphoma & $(70)$ \\
\hline & TGF- $\beta 1$ (shRNA) & Lymphoma & $(44,71)$ \\
\hline & CD21 (Gp350) & CLL & $(72)$ \\
\hline & Apoptosis (Apo2L/TRAIL) & MM & (73) \\
\hline
\end{tabular}

CLL, chronic lymphocytic leukemia; DLBCL, diffuse large B-cell lymphoma; MM, multiple myeloma; ALL, lymphocytic leukemia; HL, Hodgkin lymphoma.

complexes. Impairing the pro-tumoral effect of $\mathrm{EV}$ can be achieved by acting through two main axes. On one hand, the autocrine signals, where EV bind to and modify neoplastic cells themselves, could be disrupted (41). On the other hand, the role of EV in the cross-talk between cancer cells and the supportive microenvironment could be targeted. This can have a major impact on decreasing tumor survival, proliferation, and migration (42).

In B-cell malignancies, Koch and colleagues demonstrated that treating several diffuse large B-cell lymphoma (DLBCL) cell lines with indomethacin, a non-steroidal anti-inflammatory drug, suppressed EV release and strongly decreased tumor progression. They further demonstrated that a decreased EV release guarantees a more potent effect of cytostatic drugs, such as anthracyclines and anthracenediones, both in vitro and in vivo (42). Another strategy implies the reduction of EV uptake by blocking key factors, e.g., heparan sulfate proteoglycans (HSPGs), on target cells (41). HSPGs have been suggested to act as a receptor for EV internalization. In line with this, we demonstrated that pre-treatment of EV with low molecular weight heparin, a HS analog, strongly decreases the uptake of CLL-derived EV by target cells (30).

Several studies have demonstrated how EV have a strong impact on the immune system, allowing a more efficient tumor immune evasion (13). One of the characteristics associated with this phenomenon is the presence of immune checkpoint (ICP) ligands on tumor-derived EV. Chen et al. (43) recently showed an increase in PD-L1 molecule on melanoma derivedEV upon interferon- $\gamma$ stimulation. Considering that PD-L1 and, potentially, other ICP molecules are present on EV derived from B-cell malignancies characterized by a strong immunesuppressed microenvironment (e.g., CLL), therapies such as recombinant blocking antibodies can represent an effective solution in targeting immune-suppressive EV. The same strategy can also be extended to other EV molecules; for instance, targeting leukemia-derived EV bearing transforming growth factor- $\beta 1$ (TGF- $\beta 1$ ) has been shown to improve anti-leukemia immunity (44).

Molecules carried by tumor-derived EV are not the only source of harming. EV released by tumor microenvironment B-cells carry CD39 and CD73, two surface molecules able to hydrolyze ATP released by dying cancer cells to adenosine and effectively hijack CD8 $\mathrm{T}$ cell immune activity by binding the A2A adenosine receptors (45). In line with this, it is possible to speculate that B-cell malignancy-derived EV may have a similar effect, if not greater, given their higher concentration in the tumor milieu. The study of Zhang et al. (45) shows that a decrease of B-cell-derived EV bearing CD73 and CD39 can be achieved by deregulating the docking protein RAB27A. This has been performed using an inactivated Epstein-Barr virusmediated siRNA, but it is also possible to generate EV (e.g., derived from modified stroma cell lines) carrying RAB27A siRNA and specifically deliver it to tumor cells.

Targeting B-cell malignancy EV represents an interesting strategy in the treatment of cancer. These approaches imply obvious risks and limitations, such as possible drug resistance and off-targets, which can ultimately lead to decreased efficacy of the therapy.

\section{Role of EV in Immune Evasion and Use in Immunotherapy}

The immune system is one of the main players involved in cancer cell recognition and elimination (46-48). However, cancers deploy numerous strategies to repress the immune system during disease development and, thus, effectively evade immune surveillance. The concept behind immunotherapy is to re-activate the patient's immune system in order to recognize and remove cancer cells. One example of the many mechanisms involved in immunoevasion is the use of EV to fully re-educate 
the immune microenvironment, causing a domino effect and ultimately leading to overall immune repression and cancer development (13).

Being released by neoplastic cells, EV represent an important source of selective antigens that educate naïve immune cells (49, 50). As for solid tumors (51-53), leukemia-derived EV are loaded with antigens and several immunogenic molecules, such as TGF$\beta$ and IL-6, capable to impair dendritic cells (DCs) to build a specialized immune response against neoplastic cells (54-57). EV structure and characteristics allow them to specifically drive DC-dependent immunization; in fact, stimulation of DCs with only leukemic cell lysate results in failure to build an appropriate immune response $(55,56)$.

Immunotherapy can also be used in parallel with chemotherapy. Guo and colleagues combined leukemia-specific DC-derived EV with cyclophosphamide and polyinosinicpolycytidylic acid sodium salt (poly I:C) (58). This combination is based on the ability of DC-derived EV to stimulate $\mathrm{T}$ lymphocyte proliferation and enhance their cytotoxic activity against leukemia, while poly I:C acts on DC maturation. This strategy led to leukemia cell suppression in vitro and increased survival of tumor-bearing mice (58).

Targeting tumor-derived EV surface molecules is also a valuable strategy to strongly reduce their activity on immune cells. As we have previously mentioned, TGF- $\beta 1$ is enriched in $\mathrm{EV}$ released by a wide range of tumors and, acting on various immune cells such as DCs, is one of the main causes of immune escape (57). EV released by acute lymphocytic leukemia (ALL) L1210 cell line carry high levels of TGF- $\beta 1$. By using small hairpin RNA (shRNA), Huang and colleagues knocked down TGF- $\beta 1$ in the ALL cell line, thus removing it from their EV and reconstituting DC maturation and activity in vivo. Additionally, the same group demonstrated that pulsed DCs were able to increase $\mathrm{T}$ cell proliferation and enhance cytotoxic activity against ALL cells (44).

Dendritic cells are not the only immune cells that can be used to re-establish a proper cytotoxic activity against B-cell malignancies. Another valid target is natural killer (NK) cells, whose activity is repressed by molecules, such as NKG2D ligands, exposed on hematological malignancy (HM)-derived EV $(59,60)$. One particular study presented a double role of the NKp30 ligand BAG6 in CLL. First, high levels of soluble BAG6 can be detected in the plasma of CLL patients, being one of the causes for the defect in NK cytotoxicity observed in these patients (61). At the same time, BAG6 is released via EV by healthy cells in response to cellular stress and has a protective activity by enhancing NK cytotoxicity (60). Based on this, it is possible to hypothesize that treatment with BAG6 ${ }^{+}$EV derived from stressed cells may be a reliable opportunity to maintain (and possibly to re-establish) NK activity during cancer development.

Despite their evident activation effects on the immune system, the use of EV-based vaccination is still under discussion, although several studies already suggest how EV can be used to build an effective immune response before tumor cells arise. In line with this, DC cells pulsed with DLBCL-derived EV have been shown to stimulate T lymphocyte expansion and, by consequence, increase anti-lymphoma immunity in mice (62). These results are in accordance with other studies where leukemia-derived EV can be used to build anti-leukemia immunity, with evident results both in vitro and in vivo $(54,63)$. Finally, Qazi and colleagues showed that EV antigens alone are enough to induce memory $\mathrm{T}$ lymphocytes through B-cell activation (64).

Altogether these findings position $\mathrm{EV}$ as potent immune modulators that can become a major tool for immunotherapy and vaccine design.

\section{Engineered EV}

The ability of EV to bind to specific receptors on both tumor and microenvironment cells through surface molecules makes them an interesting tool to transfer exogenous cytotoxic and inhibitory molecules for therapeutic purposes (65). Furthermore, EV represent a useful method to deliver anti-tumor drugs due to their ability to retain stable concentrations of the components loaded in them as well as a natural accumulation in vascular sites, such as inflammation and wounds, tumor, and infection areas (66-69).

Lunavat and colleagues successfully engineered EV-like nanoparticles (NV) that contain siRNA and showed that silencing c-Myc by this approach efficiently activates poly (ADP-ribose) polymerase-dependent apoptotic pathways in treated $\lambda 820$ lymphoma cells (70). More recently, shRNA strategy has been used to silence TGF- $\beta 1$ in lymphoma cells, forcing them to release TGF- $\beta 1$-depleted EV. By removing this strong antitumorimmune surveillance inhibitor, the authors achieved an increase in the response of the immune system against leukemic cells (71). In line with this, engineered EV were also used to transfer specific antigens, with the purpose of re-activating or enhancing the immune system. Stromal cells transfected with the Epstein-Barr virus (EBV) protein gp350 release gp350 ${ }^{+} \mathrm{EV}$ that specifically interacts with CD21 on B-cells. Gp350 ${ }^{+} \mathrm{EV}$ were further engineered to carry CD154. By treating CLL patients' cells, the authors achieved the internalization of EV in leukemic cells and a strong immunogenic effect, leading to a dual activation of tumor-associated and EBV-specific T cells (72).

Further strategies based on EV-like nanostructures have been explored in B-cell malignancies. Artificial lipid vesicles (ALV) decorated with bioactive Apo2 ligand/TNF-related apoptosisinducing ligand were tested on lymphoma and MM cell lines. These novel ALV showed a consistent pro-apoptotic effect in multiple HM cell lines while sparing normal cells (such as $\mathrm{CD}^{+}$and $\mathrm{CD}^{+} \mathrm{T}$ cells) in vitro and with no sign of toxicity in vivo (73).

Engineered EV can be broadly produced in vitro using a wide range of cell lines. Mesenchymal stem cells (MSCs) have been shown to be a valuable tool for the production of EV with high tropism toward tumor cells. In line with this, MSCs can be modified to release engineered EV with various downstream applications and means (74).

As mentioned before, ICPs on EV surface are important targets for immunotherapy. As alternative to monoclonal antibodies, EV released by modified MSCs can be used as antagonists for delivering immune checkpoint blockade proteins (e.g., ICP receptors or monoclonal antibodies) (75). A further well-known immune escape strategy applied by tumors is the 
overexpression of CD47, also carried by tumor-derived EV, which interacts with the signal regulatory protein $\alpha$ (SIRP $\alpha)$ on phagocytic cells, limiting their activity (76). The production of EV bearing SIRP $\alpha$ molecules has been shown to successfully hamper the tumor "don't-eat-me" signal in favor to macrophage phagocytosis (77).

Recently, a new class of engineered EV has been tested, with the aim to enhance $\mathrm{T}$ cell activity against tumor cells. Synthetic multivalent antibodies retargeted (SMART) EV bear monoclonal antibodies against $\mathrm{CD} 3$ and cancer cell-associated epidermal growth factor receptor (EGFR). These characteristics allow them to act as a bridge directing $\mathrm{T}$ cytotoxic cells toward tumor cells, thus inducing crosstalk and enhancing antitumor response (78). SMART EV may have a potential application on MM due to its high level of EGFR, which guarantee proliferation and resistance to conventional therapies $(79,80)$. Furthermore, the same strategy could be applied for other B-cell malignancies, such as CLL and lymphoma, by targeting highly enriched surface molecules.

$\mathrm{EV}$ are also able to epigenetically reprogram target cells and to completely change their phenotype in a short time (81, 82). A potential strategy in the re-activation of the immune system can be to inhibit the molecules responsible for this deep change as well as creating engineered EV carrying specific cargo components (e.g., siRNA and shRNA) that are able to lift the immune cell exhaustion by rewiring the epigenetic landscape.

An interesting application for engineered EV is to use them as an instrument for decoy of cytokines (e.g., proinflammatory cytokines). This strategy actively restrains tumor effect on microenvironment cells (e.g., reducing inflammation). An example comes from a recent preprint where stroma-derived EV were engineered to bear tumor necrosis factor receptor 1 and interleukin 6-signal transducer. The authors reported striking effects concerning inflammation reduction and survival of experimental mouse models (83). A possible application of this strategy in B-cell malignancies would be to decoy important cytokines related to immune suppression, such as IL-10 and TGF- $\beta 1(44,84)$.

Various studies have successfully used EV as a drug delivery system. The rationale to generate EV containing specific drugs is based on cell ability to encapsulate exogenous material and release either actively (e.g., microvesicles budding) or by cellular death consequence (apoptotic bodies). Based on this, EV released by drug-treated tumor cells have been used to deliver specific chemotherapeutic agents to untreated tumor cells (85). EVbased drug delivery has been tested on various cancer models, including multiple drug resistance (MDR). In each case, EV shielded the therapeutic agents and delivered them to tumor cells, demonstrating a higher cytotoxic effect compared with its administration alone $(85,86)$. Furthermore, Osterman et al. (87) have shown how EV can be used to encapsulate toxic drugs, such as curcumin, and be specifically directed to cancer cells, highly reducing any side effect. Despite the fact that no EV-based drug delivery has been established for B-cell malignancies, strategies to encapsulate chemotherapeutic agents should be explored. One of the many possibilities could be to encapsulate the ceramide supplement $\mathrm{C} 6$ in EV to target MM cells. Indeed Chang and colleagues showed in vitro how C6 ceramide treatment leads to increased apoptosis and block the proliferation of $\mathrm{MM}$ cells by upregulating miRNA clusters such as miR-202 and miR-16 (88).

Engineered EV, as well as artificial $\mathrm{NV}$, are powerful and plastic tools to be deployed against B-cell malignancies. As mentioned above, it is evident that one of the multiple advantages lies in the possibility to combine this strategy with other well-established approaches, such as those aiming at reactivating the host immune system. Furthermore, using engineered EV with a tropism toward specific cells may improve the targeting of tumor EV. Loading shRNA into these delivery systems has the potential to reduce tumor EV production, release, and uptake. Finally, it is important to consider the high affinity of engineered EV for tumor cells as a strategic tool to deliver chemotherapy drugs, thus reducing any off-target side effects.

\section{ONGOING CLINICAL TRIAL USING EV}

Several clinical trials involving EV are currently ongoing. In cancer, the vast majority are applied to solid tumors. The aims are various: to establish novel sources and standardized methods to isolate EV from patients (NCT03821909), to deepen the characterization of cancer $\mathrm{EV}$ as predictive biomarkers (NCT03830619), to develop EV-based vaccination (NCT01159288), and finally to further improve EV-based treatment (NCT03608631).

In reference to B-cell malignancies, the ExoReBly project (NCT03985696) has the overall aim to characterize DLBCLderived EV from patients' samples. The rationale of this clinical trial is based on the fact that half of the patients subjected to immunotherapy-typically with the aim to stimulate immune activity against $\mathrm{CD} 20$-fails to show any benefit. The major hypothesis is that the presence of a high amount of CD20 and PD-L1, present on DLBCL-derived EV, acts as a decoy target for rituximab antibody and as a strong immunosuppressive signal, respectively, leading to therapeutic resistance. Apart from the characterization of DLBCL-derived EV, the project aims to use these $\mathrm{EV}$ as a marker of response to therapy and disease outcome. Interestingly, we made a similar observation with CLL-derived EV presenting high levels of CD20 on their surface and being able to act as a decoy for rituximab, potentially highlighting a conserved mechanism among B-cell malignancies (30).

With over 70 clinical trials, EV are gaining more attention in view of practical and clinical applications. A small percentage of these clinical trials take in consideration HM, and only one is being developed in B-cell malignancies. Nevertheless, every year, new information concerning EV impact on cancer progression is generated, which strongly suggests that more applications for HM will arise.

\section{CONCLUSION}

The path to decipher the complex characteristics of EV composition in B-cell malignancies, as well as the biological role that they accomplish in the behavior of tumors, has shown 
important advances in the last decade. The increasing amount of novel data recently generated reflects a continuous interest for studying EV within the community, due in part to their potential as a tool to improve cancer diagnosis and therapy. It is now well established that EV can mirror the tumor cells they originate from and can carry key targetable molecules strongly related to cancer biology, like immune checkpoints among many others. Consequently, several additional important discoveries herein summarized pinpoint a central role attributed to EV in B-cell driven diseases diagnosis and follow-up and for putative novel treatment strategies.

However, these reports remain in the area of translational research but are not yet successfully translated to clinical applications for patients with B-cell malignancies. While the treatment of solid cancers using an EV-derived rationale is already ongoing in advanced clinical trial phases, this is neither the case for the diagnosis and the follow-up nor for any treatment in B-cell-originated neoplastic diseases. The fact that EV-based approaches work properly in other chronic neoplastic and nonneoplastic diseases represents a motivation to fully enhance

\section{REFERENCES}

1. Raposo G, Stoorvogel W. Extracellular vesicles: exosomes, microvesicles, and friends. J Cell Biol. (2013) 200:373-83. doi: 10.1083/jcb.201211138

2. Lotvall J, Hill AF, Hochberg F, Buzas EI, Di Vizio D, Gardiner C, et al. Minimal experimental requirements for definition of extracellular vesicles and their functions: a position statement from the International Society for Extracellular Vesicles. J Extracell Vesicles. (2014) 3:26913. doi: 10.3402/jev.v3.26913

3. Thery C, Witwer KW, Aikawa E, Alcaraz MJ, Anderson JD, Andriantsitohaina R, et al. Minimal information for studies of extracellular vesicles 2018 (MISEV2018): a position statement of the International Society for Extracellular Vesicles and update of the MISEV2014 guidelines. J Extracell Vesicles. (2018) 7:1535750. doi: 10.1080/20013078.2018.1535750

4. Witwer KW, Soekmadji C, Hill AF, Wauben MH, Buzas EI, Di Vizio D, et al. Updating the MISEV minimal requirements for extracellular vesicle studies: building bridges to reproducibility. J Extracell Vesicles. (2017) 6:1396823. doi: 10.1080/20013078.2017.1396823

5. Wierz M, Pierson S, Gargiulo E, Guerin C, Moussay E, Paggetti J. Purification of Leukemia-derived exosomes to study microenvironment modulation. Methods Mol Biol. (2019) 1884:231-45. doi: 10.1007/978-1-4939-8885-3_16

6. Keller S, Ridinger J, Rupp AK, Janssen JW, Altevogt P. Body fluid derived exosomes as a novel template for clinical diagnostics. J Transl Med. (2011) 9:86. doi: 10.1186/1479-5876-9-86

7. Pan BT, Johnstone RM. Fate of the transferrin receptor during maturation of sheep reticulocytes in vitro: selective externalization of the receptor. Cell. (1983) 33:967-78. doi: 10.1016/0092-8674(83)90040-5

8. Keerthikumar S, Chisanga D, Ariyaratne D, Al Saffar H, Anand S, Zhao K, et al. ExoCarta: a web-based compendium of exosomal cargo. J Mol Biol. (2016) 428:688-92. doi: 10.1016/j.jmb.2015.09.019

9. Puhm F, Afonyushkin T, Resch U, Obermayer G, Rohde M, Penz T, et al. Mitochondria are a subset of extracellular vesicles released by activated monocytes and induce type I IFN and TNF responses in endothelial cells. Circ Res. (2019) 125:43-52. doi: 10.1161/CIRCRESAHA.118.314601

10. Hough KP, Trevor JL, Strenkowski JG, Wang Y, Chacko BK, Tousif S, et al. Exosomal transfer of mitochondria from airway myeloid-derived regulatory cells to T cells. Redox Biol. (2018) 18:54-64. doi: 10.1016/j.redox.2018.06.009

11. Xu R, Rai A, Chen M, Suwakulsiri W, Greening DW, Simpson RJ. Extracellular vesicles in cancer - implications for future improvements in cancer care. Nat Rev Clin Oncol. (2018) 15:617-38. doi: 10.1038/s41571-018-0036-9

12. Wang SE. Extracellular vesicles and metastasis. Cold Spring Harb Perspect Med. (2019) 10:a037275. doi: 10.1101/cshperspect.a037275 initiatives that use this rationale in HM in the near future. Thus, the upcoming decade will hopefully shed light into the clinical applicability of EV as a powerful tool for patients with B-cell neoplastic disorders.

\section{AUTHOR CONTRIBUTIONS}

EG wrote the manuscript and created the figure. PM and AL helped in writing the manuscript. EM and JP finalized the manuscript and supervised the team. All authors contributed to the article and approved the submitted version.

\section{FUNDING}

This work was supported by grants from FNRS “Télévie” to PM and AL (7.8506.19, 7.4502.17, and 7.4503.19) and from FNR Luxembourg to EG and JP (PRIDE15/10675146/CANBIO and INTER/DFG/16/11509946).

13. Gargiulo E, Paggetti J, Moussay E. Hematological malignancy-derived small extracellular vesicles and tumor microenvironment: the art of turning foes into friends. Cells. (2019) 8:511. doi: 10.3390/cells8050511

14. Haderk F, Schulz R, Iskar M, Cid LL, Worst T, Willmund KV, et al. Tumorderived exosomes modulate PD-L1 expression in monocytes. Sci Immunol. (2017) 2:eaah5509. doi: 10.1126/sciimmunol.aah5509

15. Boyiadzis $M$, Whiteside TL. Information transfer by exosomes: a new frontier in hematologic malignancies. Blood Rev. (2015) 29:281-90. doi: 10.1016/j.blre. 2015.01.004

16. Whiteside TL. Extracellular vesicles isolation and their biomarker potential: are we ready for testing? Ann Transl Med. (2017) 5:54. doi: 10.21037/atm.2017. 01.62

17. Alix-Panabieres C, Schwarzenbach H, Pantel K. Circulating tumor cells and circulating tumor DNA. Annu Rev Med. (2012) 63:199-215. doi: 10.1146/ annurev-med-062310-094219

18. Komatsubara KM, Sacher AG. Circulating tumor DNA as a liquid biopsy: current clinical applications and future directions. Oncology (Williston Park). (2017) 31:618-27.

19. Diaz LA Jr., Bardelli A. Liquid biopsies: genotyping circulating tumor DNA. J Clin Oncol. (2014) 32:579-86. doi: 10.1200/JCO.2012.45.2011

20. Sedlackova T, Repiska G, Celec P, Szemes T, Minarik G. Fragmentation of DNA affects the accuracy of the DNA quantitation by the commonly used methods. Biol Proced Online. (2013) 15:5. doi: 10.1186/1480-9222-15-5

21. Ignatiadis M, Lee M, Jeffrey SS. Circulating tumor cells and circulating tumor DNA: challenges and opportunities on the path to clinical utility. Clin Cancer Res. (2015) 21:4786-800. doi: 10.1158/1078-0432.CCR-14-1190

22. Boukouris S, Mathivanan S. Exosomes in bodily fluids are a highly stable resource of disease biomarkers. Proteomics Clin Appl. (2015) 9:358-67. doi: 10.1002/prca.201400114

23. Abels ER, Breakefield XO. Introduction to extracellular vesicles: biogenesis, RNA Cargo selection, content, release, and uptake. Cell Mol Neurobiol. (2016) 36:301-12. doi: 10.1007/s10571-016-0366-Z

24. Caivano A, Laurenzana I, De Luca L, La Rocca F, Simeon V, Trino S, et al. High serum levels of extracellular vesicles expressing malignancy-related markers are released in patients with various types of hematological neoplastic disorders. Tumour Biol. (2015) 36:9739-52. doi: 10.1007/s13277-0153741-3

25. Ghosh AK, Secreto CR, Knox TR, Ding W, Mukhopadhyay D, Kay NE. Circulating microvesicles in B-cell chronic lymphocytic leukemia can stimulate marrow stromal cells: implications for disease progression. Blood. (2010) 115:1755-64. doi: 10.1182/blood-2009-09-242719 
26. Caivano A, La Rocca F, Laurenzana I, Trino S, De Luca L, Lamorte D, et al. Extracellular vesicles in hematological malignancies: from biology to therapy. Int J Mol Sci. (2017) 18:1183. doi: 10.3390/ijms18061183

27. Siravegna G, Marsoni S, Siena S, Bardelli A. Integrating liquid biopsies into the management of cancer. Nat Rev Clin Oncol. (2017) 14:531-48. doi: 10.1038/ nrclinonc.2017.14

28. Hong CS, Muller L, Whiteside TL, Boyiadzis M. Plasma exosomes as markers of therapeutic response in patients with acute myeloid leukemia. Front Immunol. (2014) 5:160. doi: 10.3389/fimmu.2014.00160

29. Moussay E, Wang K, Cho JH, van Moer K, Pierson S, Paggetti J, et al. MicroRNA as biomarkers and regulators in B-cell chronic lymphocytic leukemia. Proc Natl Acad Sci USA. (2011) 108:6573-8. doi: 10.1073/pnas. 1019557108

30. Paggetti J, Haderk F, Seiffert M, Janji B, Distler U, Ammerlaan W, et al. Exosomes released by chronic lymphocytic leukemia cells induce the transition of stromal cells into cancer-associated fibroblasts. Blood. (2015) 126:1106-17. doi: 10.1182/blood-2014-12-618025

31. Yeh YY, Ozer HG, Lehman AM, Maddocks K, Yu L, Johnson AJ, et al. Characterization of CLL exosomes reveals a distinct microRNA signature and enhanced secretion by activation of BCR signaling. Blood. (2015) 125:3297305. doi: 10.1182/blood-2014-12-618470

32. van Eijndhoven MA, Zijlstra JM, Groenewegen NJ, Drees EE, van Niele S, Baglio SR, et al. Plasma vesicle miRNAs for therapy response monitoring in Hodgkin lymphoma patients. JCI Insight. (2016) 1:e89631. doi: 10.1172/jci. insight. 89631

33. Manier S, Liu CJ, Avet-Loiseau H, Park J, Shi J, Campigotto F, et al. Prognostic role of circulating exosomal miRNAs in multiple myeloma. Blood. (2017) 129:2429-36. doi: 10.1182/blood-2016-09-742296

34. Brinkman K, Meyer L, Bickel A, Enderle D, Berking C, Skog J, et al. Extracellular vesicles from plasma have higher tumour RNA fraction than platelets. J Extracell Vesicles. (2020) 9:1741176. doi: 10.1080/20013078.2020. 1741176

35. Pugholm LH, Baek R, Sondergaard EK, Revenfeld AL, Jorgensen MM, Varming K. Phenotyping of leukocytes and leukocyte-derived extracellular vesicles. J Immunol Res. (2016) 2016:6391264. doi: 10.1155/2016/63 91264

36. De Luca L, D’Arena G, Simeon V, Trino S, Laurenzana I, Caivano A, et al. Characterization and prognostic relevance of circulating microvesicles in chronic lymphocytic leukemia. Leuk Lymphoma. (2017) 58:1424-32. doi: 10. 1080/10428194.2016.1243790

37. Belov L, Matic KJ, Hallal S, Best OG, Mulligan SP, Christopherson RI. Extensive surface protein profiles of extracellular vesicles from cancer cells may provide diagnostic signatures from blood samples. J Extracell Vesicles. (2016) 5:25355. doi: 10.3402/jev.v5.25355

38. Boysen J, Nelson M, Magzoub G, Maiti GP, Sinha S, Goswami M, et al. Dynamics of microvesicle generation in B-cell chronic lymphocytic leukemia: implication in disease progression. Leukemia. (2017) 31:350-60. doi: 10.1038/ leu.2016.217

39. Bjorklund CC, Baladandayuthapani V, Lin HY, Jones RJ, Kuiatse I, Wang H, et al. Evidence of a role for CD44 and cell adhesion in mediating resistance to lenalidomide in multiple myeloma: therapeutic implications. Leukemia. (2014) 28:373-83. doi: 10.1038/leu.2013.174

40. Harshman SW, Canella A, Ciarlariello PD, Agarwal K, Branson OE, Rocci A, et al. Proteomic characterization of circulating extracellular vesicles identifies novel serum myeloma associated markers. J Proteomics. (2016) 136:89-98. doi: 10.1016/j.jprot.2015.12.016

41. Christianson HC, Svensson KJ, van Kuppevelt TH, Li JP, Belting M. Cancer cell exosomes depend on cell-surface heparan sulfate proteoglycans for their internalization and functional activity. Proc Natl Acad Sci USA. (2013) 110:17380-5. doi: 10.1073/pnas.1304266110

42. Koch R, Demant M, Aung T, Diering N, Cicholas A, Chapuy B, et al. Populational equilibrium through exosome-mediated Wnt signaling in tumor progression of diffuse large B-cell lymphoma. Blood. (2014) 123:2189-98. doi: 10.1182/blood-2013-08-523886

43. Chen G, Huang AC, Zhang W, Zhang G, Wu M, Xu W, et al. Exosomal PD-L1 contributes to immunosuppression and is associated with anti-PD-1 response. Nature. (2018) 560:382-6. doi: 10.1038/s41586-018-0392-8
44. Huang F, Wan J, Hao S, Deng X, Chen L, Ma L. TGF-betal-silenced leukemia cell-derived exosomes target dendritic cells to induce potent anti-leukemic immunity in a mouse model. Cancer Immunol Immunother. (2017) 66:132131. doi: 10.1007/s00262-017-2028-5

45. Zhang F, Li R, Yang Y, Shi C, Shen Y, Lu C, et al. Specific decrease in B-cell-derived extracellular vesicles enhances post-chemotherapeutic CD8(+) T cell responses. Immunity. (2019) 50:738-50.e7. doi: 10.1016/j.immuni.2019. 01.010

46. Burnet FM. Immunological surveillance in neoplasia. Transplant Rev. (1971) 7:3-25. doi: 10.1111/j.1600-065x.1971.tb00461.x

47. Burnet M. Cancer; a biological approach. I. The processes of control. Br Med J. (1957) 1:779-86. doi: 10.1136/bmj.1.5022.779

48. Burnet M. Immunological factors in the process of carcinogenesis. Br Med Bull. (1964) 20:154-8. doi: 10.1093/oxfordjournals.bmb.a070310

49. Burrello J, Monticone S, Gai C, Gomez Y, Kholia S, Camussi G. Stem cellderived extracellular vesicles and immune-modulation. Front Cell Dev Biol. (2016) 4:83. doi: 10.3389/fcell.2016.00083

50. Bruno S, Deregibus MC, Camussi G. The secretome of mesenchymal stromal cells: role of extracellular vesicles in immunomodulation. Immunol Lett. (2015) 168:154-8. doi: 10.1016/j.imlet.2015.06.007

51. Andre F, Schartz NE, Movassagh M, Flament C, Pautier P, Morice P, et al. Malignant effusions and immunogenic tumour-derived exosomes. Lancet. (2002) 360:295-305. doi: 10.1016/S0140-6736(02)09552-1

52. Huang SH, Li Y, Zhang J, Rong J, Ye S. Epidermal growth factor receptorcontaining exosomes induce tumor-specific regulatory T cells. Cancer Invest. (2013) 31:330-5. doi: 10.3109/07357907.2013.789905

53. Ning Y, Shen K, Wu Q, Sun X, Bai Y, Xie Y, et al. Tumor exosomes block dendritic cells maturation to decrease the $\mathrm{T}$ cell immune response. Immunol Lett. (2018) 199:36-43. doi: 10.1016/j.imlet.2018. 05.002

54. Yao Y, Wang C, Wei W, Shen C, Deng X, Chen L, et al. Dendritic cells pulsed with leukemia cell-derived exosomes more efficiently induce antileukemic immunities. PLoS One. (2014) 9:e91463. doi: 10.1371/journal.pone.00 91463

55. Gu X, Erb U, Buchler MW, Zoller M. Improved vaccine efficacy of tumor exosome compared to tumor lysate loaded dendritic cells in mice. Int J Cancer. (2015) 136:E74-84. doi: 10.1002/ijc.29100

56. Shen C, Hao SG, Zhao CX, Zhu J, Wang C. Antileukaemia immunity: effect of exosomes against NB4 acute promyelocytic leukaemia cells. J Int Med Res. (2011) 39:740-7. doi: 10.1177/147323001103900305

57. Yang C, Kim SH, Bianco NR, Robbins PD. Tumor-derived exosomes confer antigen-specific immunosuppression in a murine delayed-type hypersensitivity model. PLoS One. (2011) 6:e22517. doi: 10.1371/journal.pone. 0022517

58. Guo F, Chang CK, Fan HH, Nie XX, Ren YN, Liu YY, et al. Antitumour effects of exosomes in combination with cyclophosphamide and polyinosinic-polycytidylic acid. J Int Med Res. (2008) 36:1342-53. doi: 10.1177/ 147323000803600623

59. Hedlund M, Nagaeva O, Kargl D, Baranov V, Mincheva-Nilsson L. Thermaland oxidative stress causes enhanced release of NKG2D ligand-bearing immunosuppressive exosomes in leukemia/lymphoma T and B cells. PLoS One. (2011) 6:e16899. doi: 10.1371/journal.pone.0016899

60. Reiners KS, Topolar D, Henke A, Simhadri VR, Kessler J, Sauer M, et al. Soluble ligands for NK cell receptors promote evasion of chronic lymphocytic leukemia cells from NK cell anti-tumor activity. Blood. (2013) 121:3658-65. doi: 10.1182/blood-2013-01-476606

61. Veuillen C, Aurran-Schleinitz T, Castellano R, Rey J, Mallet F, Orlanducci F, et al. Primary B-CLL resistance to NK cell cytotoxicity can be overcome in vitro and in vivo by priming NK cells and monoclonal antibody therapy. J Clin Immunol. (2012) 32:632-46. doi: 10.1007/s10875-011-9624-5

62. Chen Z, You L, Wang L, Huang X, Liu H, Wei JY, et al. Dual effect of DLBCL-derived EXOs in lymphoma to improve DC vaccine efficacy in vitro while favor tumorgenesis in vivo. J Exp Clin Cancer Res. (2018) 37:190. doi: 10.1186/s13046-018-0863-7

63. Bu N, Li QL, Feng Q, Sun BZ. Immune protection effect of exosomes against attack of L1210 tumor cells. Leuk Lymphoma. (2006) 47:913-8. doi: 10.1080/ 10428190500376191 
64. Qazi KR, Gehrmann U, Domange Jordo E, Karlsson MC, Gabrielsson S. Antigen-loaded exosomes alone induce Th1-type memory through a B-celldependent mechanism. Blood. (2009) 113:2673-83. doi: 10.1182/blood-200804- 153536

65. Vader P, Breakefield XO, Wood MJ. Extracellular vesicles: emerging targets for cancer therapy. Trends Mol Med. (2014) 20:385-93. doi: 10.1016/j.molmed. 2014.03.002

66. Allen TM, Newman MS, Woodle MC, Mayhew E, Uster PS. Pharmacokinetics and anti-tumor activity of vincristine encapsulated in sterically stabilized liposomes. Int J Cancer. (1995) 62:199-204. doi: 10.1002/ijc.2910620215

67. Hosseinkhani B, Kuypers S, van den Akker NMS, Molin DGM, Michiels L. Extracellular vesicles work as a functional inflammatory mediator between vascular endothelial cells and immune cells. Front Immunol. (2018) 9:1789. doi: 10.3389/fimmu.2018.01789

68. Than UTT, Guanzon D, Leavesley D, Parker T. Association of extracellular membrane vesicles with cutaneous wound healing. Int J Mol Sci. (2017) 18:956. doi: 10.3390/ijms18050956

69. Maacha S, Bhat AA, Jimenez L, Raza A, Haris M, Uddin S, et al. Extracellular vesicles-mediated intercellular communication: roles in the tumor microenvironment and anti-cancer drug resistance. Mol Cancer. (2019) 18:55. doi: 10.1186/s12943-019-0965-7

70. Lunavat TR, Jang SC, Nilsson L, Park HT, Repiska G, Lasser C, et al. RNAi delivery by exosome-mimetic nanovesicles - implications for targeting c-Myc in cancer. Biomaterials. (2016) 102:231-8. doi: 10.1016/j.biomaterials.2016.06. 024

71. Huang F, Wan J, Hu W, Hao S. Enhancement of anti-leukemia immunity by leukemia-derived exosomes via downregulation of TGF-beta1 expression. Cell Physiol Biochem. (2017) 44:240-54. doi: 10.1159/000484677

72. Ruiss R, Jochum S, Mocikat R, Hammerschmidt W, Zeidler R. EBV-gp350 confers B-cell tropism to tailored exosomes and is a neo-antigen in normal and malignant B cells-a new option for the treatment of B-CLL. PLoS One. (2011) 6:e25294. doi: 10.1371/journal.pone.0025294

73. De Miguel D, Basanez G, Sanchez D, Malo PG, Marzo I, Larrad L, et al. Liposomes decorated with Apo2L/TRAIL overcome chemoresistance of human hematologic tumor cells. Mol Pharm. (2013) 10:893-904. doi: 10.1021/ mp300258c

74. Dostert G, Mesure B, Menu P, Velot E. How do mesenchymal stem cells influence or are influenced by microenvironment through extracellular vesicles communication? Front Cell Dev Biol. (2017) 5:6. doi: 10.3389/fcell. 2017.00006

75. Yang Y, Hong Y, Cho E, Kim GB, Kim IS. Extracellular vesicles as a platform for membrane-associated therapeutic protein delivery. J Extracell Vesicles. (2018) 7:1440131. doi: 10.1080/20013078.2018.1440131

76. Chao MP, Weissman IL, Majeti R. The CD47-SIRPalpha pathway in cancer immune evasion and potential therapeutic implications. Curr Opin Immunol. (2012) 24:225-32. doi: 10.1016/j.coi.2012.01.010

77. Koh E, Lee EJ, Nam GH, Hong Y, Cho E, Yang Y, et al. Exosome-SIRPalpha, a CD47 blockade increases cancer cell phagocytosis. Biomaterials. (2017) 121:121-9. doi: 10.1016/j.biomaterials.2017.01.004
78. Cheng Q, Shi X, Han M, Smbatyan G, Lenz HJ, Zhang Y. Reprogramming exosomes as nanoscale controllers of cellular immunity. J Am Chem Soc. (2018) 140:16413-7. doi: 10.1021/jacs.8b10047

79. Chen Y, Huang R, Ding J, Ji D, Song B, Yuan L, et al. Multiple myeloma acquires resistance to EGFR inhibitor via induction of pentose phosphate pathway. Sci Rep. (2015) 5:9925. doi: 10.1038/srep09925

80. Mahtouk K, Hose D, Reme T, De Vos J, Jourdan M, Moreaux J, et al. Expression of EGF-family receptors and amphiregulin in multiple myeloma. Amphiregulin is a growth factor for myeloma cells. Oncogene. (2005) 24:351224. doi: 10.1038/sj.onc. 1208536

81. Jang YY, Collector MI, Baylin SB, Diehl AM, Sharkis SJ. Hematopoietic stem cells convert into liver cells within days without fusion. Nat Cell Biol. (2004) 6:532-9. doi: 10.1038/ncb1132

82. Landsverk HB, Hakelien AM, Kuntziger T, Robl JM, Skalhegg BS, Collas P. Reprogrammed gene expression in a somatic cell-free extract. EMBO Rep. (2002) 3:384-9. doi: 10.1093/embo-reports/kvf064

83. Gupta DGA, Conceição M, Corso G, Liang X, Seow Y, Balsu S, et al. Engineering of extracellular vesicles for display of protein biotherapeutics. bioRxiv (2020). [Preprint]. doi: 10.1101/2020.06.14.149823

84. Alhakeem SS, McKenna MK, Oben KZ, Noothi SK, Rivas JR, Hildebrandt GC, et al. Chronic Lymphocytic Leukemia-Derived IL-10 suppresses antitumor immunity. J Immunol. (2018) 200:4180-9. doi: 10.4049/jimmunol.18 00241

85. Tang K, Zhang Y, Zhang H, Xu P, Liu J, Ma J, et al. Delivery of chemotherapeutic drugs in tumour cell-derived microparticles. Nat Commun. (2012) 3:1282. doi: 10.1038/ncomms2282

86. Kim MS, Haney MJ, Zhao Y, Mahajan V, Deygen I, Klyachko NL, et al. Development of exosome-encapsulated paclitaxel to overcome MDR in cancer cells. Nanomedicine. (2016) 12:655-64. doi: 10.1016/j.nano.2015. 10.012

87. Osterman CJ, Lynch JC, Leaf P, Gonda A, Ferguson Bennit HR, Griffiths D, et al. Curcumin modulates pancreatic adenocarcinoma cell-derived exosomal function. PLoS One. (2015) 10:e0132845. doi: 10.1371/journal.pone.0132845

88. Cheng Q, Li X, Wang Y, Dong M, Zhan FH, Liu J. The ceramide pathway is involved in the survival, apoptosis and exosome functions of human multiple myeloma cells in vitro. Acta Pharmacol Sin. (2018) 39:561-8. doi: 10.1038/aps. 2017.118

Conflict of Interest: The authors declare that the research was conducted in the absence of any commercial or financial relationships that could be construed as a potential conflict of interest.

Copyright (c) 2020 Gargiulo, Morande, Largeot, Moussay and Paggetti. This is an open-access article distributed under the terms of the Creative Commons Attribution License (CC BY). The use, distribution or reproduction in other forums is permitted, provided the original author(s) and the copyright owner(s) are credited and that the original publication in this journal is cited, in accordance with accepted academic practice. No use, distribution or reproduction is permitted which does not comply with these terms. 